\title{
SIMPLICITY AND STABILITY OF THE FIRST EIGENVALUE OF A NONLINEAR ELLIPTIC SYSTEM
}

\author{
ABDELOUAHED EL KHALIL, SAID EL MANOUNI, AND MOHAMMED OUANAN
}

Received 8 July 2004 and in revised form 17 March 2005

We prove some properties of the first eigenvalue for the elliptic system $-\Delta_{p} u=\lambda|u|^{\alpha}|v|^{\beta} v$ in $\Omega,-\Delta_{q} v=\lambda|u|^{\alpha}|v|^{\beta} u$ in $\Omega,(u, v) \in W_{0}^{1, p}(\Omega) \times W_{0}^{1, q}(\Omega)$. In particular, the first eigenvalue is shown to be simple. Moreover, the stability with respect to $(p, q)$ is established.

\section{Introduction}

In this paper, we consider the nonlinear system

$$
\begin{aligned}
& -\Delta_{p} u=\lambda|u|^{\alpha}|v|^{\beta} v \quad \text { in } \Omega, \\
& -\Delta_{q} v=\lambda|u|^{\alpha}|v|^{\beta} u \quad \text { in } \Omega, \\
& (u, v) \in W_{0}^{1, p}(\Omega) \times W_{0}^{1, q}(\Omega),
\end{aligned}
$$

where $\Omega$ is a bounded domain in $\mathbb{R}^{N}, N \geq 1, p>1, q>1$, and $\alpha, \beta$ are real numbers satisfying

$(\mathrm{H})$

$$
\alpha>0, \beta>0, \quad \frac{\alpha+1}{p}+\frac{\beta+1}{q}=1 .
$$

Note that the system (1.1) is of two second-order elliptic equations. It is weakly coupled in the sense that interaction is present only in the "source terms," while the differential terms have only one dependent variable. The differential operator involved is the so-called $p$-Laplacian, that is, $\Delta_{p} u=\nabla \cdot\left(|\nabla u|^{p-2} \nabla u\right)$, which reduces to the ordinary Laplace operator $\Delta u$ when $p=2$. We mention that problem (1.1) arises in several fields of application. For instance, in the case where $p>2$, problem (1.1) appears in the study of non-Newtonian fluids, pseudoplastics for $1<p<2$, and in reaction-diffusion problems, flows through porous media, nonlinear elasticity, and glaciology for $p=4 / 3$. We can cite $[5,6]$, for more details.

Here, we define the first eigenvalue $\lambda_{1}(p, q)$ of $(1.1)$ as the least real parameter $\lambda$ for which both equations of $(1.1)$ have a nontrivial solution $(u, v)$ in the product Sobolev space $W_{0}^{1, p}(\Omega) \times W_{0}^{1, q}(\Omega)$, with $u \neq \equiv 0$ and $v \neq 0$. 
Several special cases of problem (1.1) have been considered in literature. For the case of the scalar equation, that is, (1.1) reduces to one equation when $p=q$ and $\beta=\alpha=$ $(p-2) / 2$, the simplicity of the first eigenvalue was apparently first proved by Lindqvist, see [10]. The radial case has been studied in [3] by de Thélin when the case of smooth domains was investigated-see, for example, [1] and the references therein.

Other problems have been considered in this direction-we refer to $[3,6,7]$.

Concerning systems of the type (1.1), a lot of papers have appeared in recent years dealing with equations involving $p$-Laplacian both in bounded and unbounded domains. In particular, de Thélin in [4] obtained the existence of the first eigenvalue $\lambda_{1}(p, q)$ by considering more real parameters $\alpha, \beta$ satisfying the condition $(\mathrm{H})$ and by considering also smooth bounded domains. The study of system (1.1) in the whole $\mathbb{R}^{N}$ was continued in [9], where the authors considered systems of the form

$$
\begin{gathered}
-\Delta_{p} u=\lambda a(x)|u|^{p-2} u+\lambda b(x)|u|^{\alpha-1}|v|^{\beta+1} u \quad \text { in } \mathbb{R}^{N}, \\
-\Delta_{q} v=\lambda d(x)|u|^{q-2} u+\lambda b(x)|u|^{\alpha+1}|v|^{\beta-1} v \quad \text { in } \mathbb{R}^{N}, \\
u, v>0, \quad \lim _{|x| \rightarrow+\infty} u(x)=\lim _{|x| \rightarrow+\infty} v(x)=0 .
\end{gathered}
$$

In particular, they gave an extension and generalized the results of [4] to unbounded domains. In both these papers, the first eigenvalue $\lambda_{1}(p, q)$ is proven to be positive and simple.

Recently, in [2], the author showed the simplicity of the first eigenvalue by extending the Saa's inequality to the whole $\mathbb{R}^{N}$. We mention that the stability question is not discussed there.

In this note, we show the uniqueness of the eigenvector corresponding to $\lambda_{1}(p, q)$, that is, $\lambda_{1}(p, q)$ is simple. In other words, positive solutions of (1.1) are unique modulo scaling. Especially, by establishing sufficient conditions and via some modifications of [2], we prove the simplicity result, see Theorem 3.2. The uniqueness result will be needed in the proof of the stability (continuity) of $\lambda_{1}(p, q)$ with respect to $(p, q)$.

On the other hand, note that the dependence with respect to the rheological exponent was studied both in the scalar $p$-Laplacian and system of two second-order equations, see, for example, $[9,11]$. Our purpose is also to extend these results to our case of system (1.1), see Theorem 4.1. More precisely, we study the stability of the ground state when the exponents $p$ and $q$ vary in the following constrained way:

$$
I_{\alpha, \beta}=\{(p, q) \in] 1+\infty[\times] 1+\infty[; p, q \text { satisfies }(\mathrm{H})\}
$$

Generally, the main difficulty lies in the fact that the appropriate Sobolev spaces $W_{0}^{1, p}(\Omega)$ and $W_{0}^{1, q}(\Omega)$ change with the exponents $p$ and $q$. Here, to overcome this obstacle, we use a local argument based only on the variational characterization of $\lambda_{1}(p, q)$ and use the fact that the underlying domain $\Omega$ in $\mathbb{R}^{N}$ has the so-called segment property (a sufficient regularity condition related to the geometry of $\Omega$ ). Contrary to irregular domains with 
$p \leq N$ or $q \leq N$, the situation

$$
\limsup _{(s, t) \rightarrow(p, q)} \lambda_{1}(s, t)<\lambda_{1}(p, q)
$$

becomes possible. An example is given in [11] in the scalar case.

The rest of this paper is organized as follows. In Section 2, we establish some definitions, basic properties, and preliminary results. In Section 3, we prove the simplicity of $\lambda_{1}$, and in the last section, we prove the stability by using the segment property lemma.

\section{Definitions and preliminaries}

We define the following functionals on $W_{0}^{1, p}(\Omega) \times W_{0}^{1, q}(\Omega)$ by

$$
\begin{gathered}
A(u, v)=\frac{\alpha+1}{p} \int_{\Omega}|\nabla u|^{p} d x+\frac{\beta+1}{q} \int_{\Omega}|\nabla v|^{q} d x, \\
B(u, v)=\int_{\Omega}|u|^{\alpha}|v|^{\beta} u v d x,
\end{gathered}
$$

where the Sobolev space $W_{0}^{1, t}(\Omega)$ is the completion of $C_{0}^{\infty}(\Omega), 1<t<+\infty$ equipped with the norm $\|\phi\|=\|\nabla \phi\|_{t}$. It is well known that $A, B \in C^{1}\left(W_{0}^{1, p}(\Omega) \times W_{0}^{1, q}(\Omega)\right)$. We will consider both equations of (1.1) in the weak sense.

2.1. Definition. We say that $\lambda \in \mathbb{R}$ is an eigenvalue of (1.1), if there exists a pair of functions $u \in W_{0}^{1, p}(\Omega)$ and $v \in W_{0}^{1, q}(\Omega)$ with $u \neq 0$ and $v \neq 0$ such that

$$
\begin{aligned}
& \int_{\Omega}|\nabla u|^{p-2} \nabla u \nabla \phi d x=\lambda \int_{\Omega}|u|^{\alpha}|v|^{\beta} v \phi d x, \\
& \int_{\Omega}|\nabla v|^{q-2} \nabla v \nabla \psi d x=\lambda \int_{\Omega}|u|^{\alpha}|v|^{\beta} u \psi d x,
\end{aligned}
$$

where $\phi \in W_{0}^{1, p}(\Omega)$ and $\psi \in W_{0}^{1, q}(\Omega)$. The pair $(u, v)$ is called an eigenvector. Observe that the solutions $(u, v)$ of $(1.1)$ correspond to the critical points of the energy functional $A$ on the set

$$
\left\{(u, v) \in W_{0}^{1, p}(\Omega) \times W_{0}^{1, q}(\Omega) ; B(u, v)=1\right\} .
$$

2.2. Basic properties. The system (1.1) possesses a first positive eigenvalue denoted by $\lambda_{1}(p, q)$ (for indicate the dependence with respect to $(p, q)$ ) obtained by the LjusternickSchnirelmann theory by minimizing the functional $A$ on the $C^{1}$-manifold defined by (2.3). So, we recall that $\lambda_{1}(p, q)$ can be variationally characterized as

$$
\lambda_{1}(p, q)=\inf \left\{A(u, v),(u, v) \in W_{0}^{1, p}(\Omega) \times W_{0}^{1, q}(\Omega) ; B(u, v)=1\right\} .
$$

According to advanced regularity result of [12], every minimizer of (2.4) belongs to $C^{1}(\Omega) \times C^{1}(\Omega)$. In addition, from the maximum principle of Vázquez, see [12], we deduce that the corresponding eigenpair of $\lambda_{1}(p, q),(u, v)$ are such that $u, v>0$. Hereafter such $(u, v)$ will be called positive eigenvector. 


\section{Simplicity and stability of a nonlinear elliptic system}

2.3. The segment property. We begin by defining a class of domains for which the boundary is smooth in order to guarantee that

$$
W^{1, p}(\Omega) \cap W_{0}^{1, s}(\Omega)=W_{0}^{1, p}(\Omega) \quad \forall s \in(1, p) .
$$

Definition 2.1. An open subset $\Omega$ of $\mathbb{R}^{N}$ is said to have the segment property if for any $x \in \partial \Omega$, there exists an open set $G_{x} \in \mathbb{R}^{N}$ with $x \in G_{x}$ and a point $y_{x}$ of $\mathbb{R}^{N} \backslash\{0\}$ such that if $z \in \bar{\Omega} \cap G_{x}$ and $t \in(0,1)$, then $z+t y_{x} \in \Omega$.

This property rules out that $\Omega$ lies on both sides on parts of its boundary. It also allows us to push the support of a function in $\Omega$ via a translation.

The following results play an important role in the proof of Theorem 3.2 (cf. [10]).

Lemma 2.2. Let $\Omega$ be a bounded domain in $\mathbb{R}^{N}$ having the segment property. If $u \in W^{1, p}(\Omega)$ $\cap W_{0}^{1, s}(\Omega)$ for some $s \in(1, p)$, then $u \in W_{0}^{1, p}(\Omega)$.

\section{Simplicity}

Before giving the main result of this section, we recall and prove the following lemma introduced in [8] which is needed below. We regive its proof for more convenience. First, we introduce

$$
\begin{aligned}
\Gamma_{p}(u, \phi)= & \int_{\Omega}|\nabla u|^{p} d x+(p-1) \int_{\Omega}|\nabla \phi|^{p}\left(\frac{|u|}{\phi}\right)^{p} d x \\
& -p \int_{\Omega}|\nabla \phi|^{p-2} \nabla \phi \nabla u\left(\frac{|u|^{p-2} u}{\phi^{p-1}}\right) d x \\
= & \int_{\Omega}|\nabla u|^{p} d x+\int_{\Omega} \frac{\Delta_{p} \phi}{\phi^{p-1}}|u|^{p} d x .
\end{aligned}
$$

Lemma 3.1. For all $(u, \phi) \in\left(W_{0}^{1, p}(\Omega) \cap C^{1, v}(\Omega)\right)^{2}$ with $\phi>0$ in $\Omega$ and $\nu \in(0,1)$, we have $\Gamma_{p}(u, \phi) \geq 0$, that is,

$$
\int_{\Omega}|\nabla u|^{p} \geq \int_{\Omega} \frac{-\Delta_{p} \phi}{\phi^{p-1}}|u|^{p}
$$

and if $\Gamma_{p}(u, \phi)=0$ there is $c \in \mathbb{R}$ such that $u=c \phi$.

Proof. By Young's inequality we have, for $\epsilon>0$,

$$
\begin{aligned}
\nabla u|\nabla \phi|^{p-2} \nabla \phi \frac{u|u|^{p-2}}{\phi^{p-1}} & \leq|\nabla u||\nabla \phi|^{p-1}\left(\frac{|u|}{\phi}\right)^{p-1} \\
& \leq \frac{\epsilon^{p}}{p}|\nabla u|^{p}+\frac{p-1}{p \epsilon^{p}}\left|\frac{u}{\phi}\right|^{p}|\nabla \phi|^{p} .
\end{aligned}
$$

Let $\epsilon=1$, we have, by integration over $\Omega$,

$$
p \int_{\Omega}|\nabla \phi|^{p-2} \nabla \phi \nabla u\left(\frac{|u|^{p-2} u}{\phi^{p-1}}\right) \leq \int_{\Omega}|\nabla u|^{p}+(p-1) \int_{\Omega}\left|\frac{u}{\phi}\right|^{p}|\nabla \phi|^{p} .
$$


Thus

$$
\Gamma_{p}(u, \phi) \geq 0
$$

On the other hand, if $\Gamma_{p}(u, \phi)=0$, then we obtain

$$
p \int_{\Omega}|\nabla \phi|^{p-2} \nabla \phi \nabla u\left(\frac{|u|^{p-2} u}{\phi^{p-1}}\right)-\int_{\Omega}|\nabla u|^{p}-(p-1) \int_{\Omega}\left|\frac{u}{\phi}\right|^{p}|\nabla \phi|^{p}=0
$$

and by choosing $\epsilon=1$ in (3.3), we get

$$
\int_{\Omega}\left\{\nabla u \nabla \phi|\nabla \phi|^{p-2} \frac{u|u|^{p-2}}{\phi^{p-1}}-|\nabla u||\nabla \phi|^{p-1}\left(\frac{|u|}{\phi}\right)^{p-1}\right\} d x=0 .
$$

By (3.6), we deduce that $|\nabla u|=|(u / \phi) \nabla \phi|$ and from (3.7), it follows that $\nabla u=\eta(u /$ $\phi) \nabla \phi$, where $|\eta|=1$. Hence $\Gamma_{p}(u, \phi)=0$ implies $\eta=1$ and $\nabla(u / \phi)=0$. Therefore, there is $c \in \mathbb{R}$ such that $u=c \phi$ and the lemma follows.

Theorem 3.2. $\lambda_{1}(p, q)$ is simple.

Proof. Let $(u, v)$ and $(\phi, \psi)$ be two eigenvectors associated to $\lambda_{1}(p, q)$ with $(u, v)$ positive (i.e., $u>0, v>0)$. Thanks to definition of $\lambda_{1}(p, q)$ and Hölder's inequality, we have

$$
\begin{aligned}
A(\phi, \psi) & =\lambda_{1}(p, q) B(\phi, \psi) \\
& \leq \lambda_{1}(p, q) \int_{\Omega} u^{\alpha+1} v^{\beta+1} \frac{|\phi|^{\alpha+1}|\psi|^{\beta+1}}{u^{\alpha+1} v^{\beta+1}} \\
& \leq \lambda_{1}(p, q) \int_{\Omega} u^{\alpha+1} v^{\beta+1}\left[\frac{\alpha+1}{p} \frac{|\phi|^{p}}{u^{p}}+\frac{\beta+1}{q} \frac{|\psi|^{q}}{v^{q}}\right] \\
& \leq \lambda_{1}(p, q) \int_{\Omega}\left[\frac{\alpha+1}{p} \frac{u^{\alpha} v^{\beta+1}}{u^{p-1}}|\phi|^{p}+\frac{\beta+1}{q} \frac{u^{\alpha+1} v^{\beta}}{v^{q-1}}|\psi|^{q}\right] \\
& \leq \frac{\alpha+1}{p} \int_{\Omega} \frac{-\Delta_{p} u}{u^{p-1}}|\phi|^{p}+\frac{\beta+1}{q} \int_{\Omega} \frac{-\Delta_{q} v}{v^{q-1}}|\psi|^{q} .
\end{aligned}
$$

Now, by Lemma 3.1, we have

$$
A(\phi, \psi)=\frac{\alpha+1}{p} \int_{\Omega} \frac{-\Delta_{p} u}{u^{p-1}}|\phi|^{p}+\frac{\beta+1}{q} \int_{\Omega} \frac{-\Delta_{q} v}{v^{q-1}}|\psi|^{q} .
$$

Thus

$$
\int_{\Omega}|\nabla \phi|^{p}=\int_{\Omega} \frac{-\Delta_{p} u}{u^{p-1}}|\phi|^{p}, \quad \int_{\Omega}|\nabla \phi|^{p}=\int_{\Omega} \frac{-\Delta_{q} v}{v^{q-1}}|\phi|^{q} .
$$

Again due to Lemma 3.1, there exist $k_{1}$ and $k_{2}$ in $\mathbb{R}$ such that $u=k_{1} \phi$ and $v=k_{2} \psi$. This ends the proof. 
1560 Simplicity and stability of a nonlinear elliptic system

\section{Stability}

THEOREM 4.1. Let $\Omega$ be a bounded domain having the segment property, then the function

$$
(p, q) \rightarrow \lambda_{1}(p, q)
$$

is continuous from $I_{\alpha, \beta}$ into $\mathbb{R}^{+}$.

Proof. Let $\left(t_{n}\right)_{n \geq 1}, t_{n}=\left(p_{n}, q_{n}\right)$ be a sequence in $I_{\alpha, \beta}$ converging to $t=(p, q)$, with $t \in I_{\alpha, \beta}$. We claim that

$$
\lim _{n \rightarrow+\infty} \lambda_{1}\left(p_{n}, q_{n}\right)=\lambda_{1}(p, q)
$$

Indeed, let $(\phi, \psi) \in C_{0}^{\infty}(\Omega) \times C_{0}^{\infty}(\Omega)$ such that $B(\phi, \psi)>0$. Then by the variational characterization of $\lambda_{1}(p, q)$, we have

$$
\lambda_{1}\left(p_{n}, q_{n}\right) \leq \frac{\left((\alpha+1) / p_{n}\right)\|\nabla \phi\|_{p_{n}}^{p_{n}}+\left((\beta+1) / q_{n}\right)\|\nabla \psi\|_{q_{n}}^{q_{n}}}{B(\phi, \psi)} .
$$

Applying the dominated convergence theorem, we find

$$
\limsup _{n \rightarrow+\infty} \lambda_{1}\left(p_{n}, q_{n}\right) \leq \frac{((\alpha+1) / p)\|\nabla \phi\|_{p}^{p}+((\beta+1) / q)\|\nabla \psi\|_{q}^{q}}{B(\phi, \psi)} .
$$

By passing to infimum of the right-hand side of (4.4), we obtain

$$
\limsup _{n \rightarrow+\infty} \lambda_{1}\left(p_{n}, q_{n}\right) \leq \lambda_{1}(p, q)
$$

Let now, $\left\{p_{n_{k}}, q_{n_{k}}\right\}_{k \geq 1}$ be a subsequence of $\left(t_{n}\right)_{n \geq 1}$ such that

$$
\lim _{k \rightarrow+\infty} \lambda_{1}\left(p_{n_{k}}, q_{n_{k}}\right)=\liminf _{n \rightarrow+\infty} \lambda_{1}\left(p_{n}, q_{n}\right)
$$

Fix $\epsilon_{0}>0$ small enough so that for all $\epsilon \in\left(0, \epsilon_{0}\right)$,

$$
\begin{gathered}
1<\min (p-\epsilon, q-\epsilon) \\
\max (p+\epsilon, q+\epsilon)<\min \left((p-\epsilon)^{*},(q-\epsilon)^{*}\right),
\end{gathered}
$$

where, for $s \in(1,+\infty), s^{*}=N s /(N-s)$ if $1<s<N$ and $s^{*}=+\infty$ if $s \geq N$. 
For each $k \in \mathbb{N}^{*}$, let $\left(u_{\left(p_{n_{k}}, q_{n_{k}}\right)}, v_{\left(p_{n_{k}}, q_{n_{k}}\right)}\right) \in W_{0}^{1, p_{n_{k}}}(\Omega) \times W_{0}^{1, q_{n_{k}}}(\Omega)$ be the first eigenvector associated to $\lambda_{1}\left(p_{n_{k}}, q_{n_{k}}\right)$, that is, $u_{\left(p_{n_{k}}, q_{n_{k}}\right)}, v_{\left(p_{n_{k}}, q_{n_{k}}\right)}>0$ with

$$
\begin{gathered}
B\left(u_{\left(p_{n_{k}}, q_{n_{k}}\right)}, v_{\left(p_{n_{k}}, q_{n_{k}}\right)}\right)=1 \\
\lambda_{1}\left(p_{n_{k}}, q_{n_{k}}\right)=\frac{\alpha+1}{p_{n_{k}}}\left\|\nabla u_{\left(p_{n_{k}}, q_{n_{k}}\right)}\right\|_{p_{n_{k}}}^{p_{n_{k}}}+\frac{\beta+1}{q_{n_{k}}}\left\|\nabla v_{\left(p_{n_{k}}, q_{n_{k}}\right)}\right\|_{q_{n_{k}}}^{q_{n_{k}}} .
\end{gathered}
$$

By using Hölder's inequality, with $\epsilon \in\left(0, \epsilon_{0}\right)$, we get simultaneously

$$
\begin{aligned}
&\left\|\nabla u_{\left(p_{n_{k}}, q_{n_{k}}\right)}\right\|_{p-\epsilon} \leq\left\|\nabla u_{\left(p_{n_{k}}, q_{n_{k}}\right)}\right\|_{p_{n_{k}}}|\Omega|^{\left(p_{n_{k}}-p+\epsilon\right) / p_{n_{k}}(p-\epsilon)}, \\
&\left\|\nabla v_{\left(p_{n_{k}}, q_{n_{k}}\right)}\right\|_{q-\epsilon} \leq\left\|\nabla v_{\left(p_{n_{k}}, q_{n_{k}}\right)}\right\|_{q_{n_{k}}}|\Omega|^{\left(q_{n_{k}}-q+\epsilon\right) / q_{n_{k}}(q-\epsilon)} .
\end{aligned}
$$

According to (4.10), we deduce

$$
\begin{aligned}
& \left\|\nabla u_{\left(p_{n_{k}}, q_{n_{k}}\right)}\right\|_{p-\epsilon} \leq\left\{\frac{p_{n_{k}} \lambda_{1}\left(p_{n_{k}}, q_{n_{k}}\right)}{\alpha+1}\right\}^{1 / p_{n_{k}}}|\Omega|^{\left(p_{n_{k}}-p+\epsilon\right) / p_{n_{k}}(p-\epsilon),} \\
& \left\|\nabla v_{\left(p_{n_{k}}, q_{n_{k}}\right)}\right\|_{q-\epsilon} \leq\left\{\frac{q_{n_{k}} \lambda_{1}\left(p_{n_{k}}, q_{n_{k}}\right)}{\beta+1}\right\}^{1 / q_{n_{k}}}|\Omega|^{\left(q_{n_{k}}-q+\epsilon\right) / q_{n_{k}}(q-\epsilon)} .
\end{aligned}
$$

Due to (4.5), it follows that $\left(\lambda_{1}\left(p_{n_{k}}, q_{n_{k}}\right)\right)_{k \geq 1}$ is a bounded sequence. Then, from (4.12) we conclude that $\left(u_{\left(p_{n_{k}}, q_{n_{k}}\right)}\right)_{k \geq 1}$ (resp., $\left.\left(v_{\left(p_{n_{k}}, q_{n_{k}}\right)}\right)_{k \geq 1}\right)$ is bounded in $W_{0}^{1, p-\epsilon}(\Omega)$ (resp., in $\left.W_{0}^{1, q-\epsilon}(\Omega)\right)$. Therefore, by compactness and (4.8), we have $u_{\left(p_{n_{k}}, q_{n_{k}}\right)} \rightarrow u$ weakly in $W_{0}^{1, p-\epsilon}(\Omega)$, strongly in $L^{p+\epsilon}(\Omega)$, and a.e in $\Omega$ (still denoted by $\mathcal{u}_{\left(p_{n_{k}}, q_{n_{k}}\right)}$ ). We have also $v_{\left(p_{n_{k}}, q_{n_{k}}\right)} \rightarrow v$ strongly in $L^{q+\epsilon}(\Omega)$ and a.e in $\Omega$ (for a subsequence if it is necessary). Clearly $u \in L^{p}(\Omega)$ and $v \in L^{q}(\Omega)$, and are independent of $\epsilon$. On the other hand, the weak lower semicontinuity of the norm implies that

$$
\begin{aligned}
\|\nabla u\|_{p-\epsilon} & \leq\left\{\frac{p}{\alpha+1}\right\}^{1 / p}\left\{\lim _{k \rightarrow+\infty} \lambda_{1}\left(p_{n_{k}}, q_{n_{k}}\right)\right\}^{1 / p}|\Omega|^{\epsilon / p(p-\epsilon)}, \\
\|\nabla v\|_{q-\epsilon} & \leq\left\{\frac{q}{\beta+1}\right\}^{1 / q}\left\{\lim _{k \rightarrow+\infty} \lambda_{1}\left(p_{n_{k}}, q_{n_{k}}\right)\right\}^{1 / q}|\Omega|^{\epsilon / q(q-\epsilon)} .
\end{aligned}
$$

Since $|\nabla u|^{p-\epsilon} \rightarrow|\nabla u|^{p}$ and $|\nabla v|^{q-\epsilon} \rightarrow|\nabla v|^{q}$ a.e on $\Omega$, as $\epsilon \rightarrow 0^{+}$, then the Fatou lemma yields with (4.13)

$$
\begin{gathered}
\|\nabla u\|_{p}^{p} \leq \frac{p}{\alpha+1} \lim _{k \rightarrow+\infty} \lambda_{1}\left(p_{n_{k}}, q_{n_{k}}\right)<+\infty, \\
\|\nabla v\|_{q}^{q} \leq \frac{q}{\beta+1} \lim _{k \rightarrow+\infty} \lambda_{1}\left(p_{n_{k}}, q_{n_{k}}\right)<+\infty .
\end{gathered}
$$

Consequently, we have $u \in W_{0}^{1, p-\epsilon}(\Omega) \cap W^{1, p}(\Omega)$ and $v \in W_{0}^{1, q-\epsilon}(\Omega) \cap W^{1, q}(\Omega)$.

This implies by Lemma 2.2 that

$$
u \in W_{0}^{1, p}(\Omega), \quad v \in W_{0}^{1, q}(\Omega) .
$$




\section{Simplicity and stability of a nonlinear elliptic system}

Finally, from (4.10), (4.11), and from the weak lower semicontinuity of the norm, it follows by letting $k$ tend to infinity that

$$
\frac{1}{|\Omega|^{\epsilon /(p-\epsilon)}} \frac{\alpha+1}{p}\|\nabla u\|_{p-\epsilon}^{p}+\frac{1}{|\Omega|^{\epsilon /(q-\epsilon)}} \frac{\beta+1}{q}\|\nabla v\|_{q-\epsilon}^{q} \leq \lim _{k \rightarrow+\infty} \lambda_{1}\left(p_{n_{k}}, q_{n_{k}}\right) .
$$

Letting now $\epsilon \rightarrow 0^{+}$, the Fatou Lemma implies

$$
\frac{\alpha+1}{p}\|\nabla u\|_{p}^{p}+\frac{\beta+1}{q}\|\nabla v\|_{q}^{q} \leq \lim _{k \rightarrow+\infty} \lambda_{1}\left(p_{n_{k}}, q_{n_{k}}\right) .
$$

Since $u \in W_{0}^{1, p}(\Omega)$ and $v \in W_{0}^{1, q}(\Omega)$, then by the variational characterization of $\lambda_{1}(p, q)$ and the simplicity of the first eigenvector, we deduce that

$$
\lambda_{1}(p, q) \leq \lim _{k \rightarrow+\infty} \lambda_{1}\left(p_{n_{k}}, q_{n_{k}}\right)=\lim _{n \rightarrow+\infty} \lambda_{1}\left(p_{n}, q_{n}\right) .
$$

Due to (4.5) and (4.18), we conclude that

$$
\lim _{n \rightarrow+\infty} \lambda_{1}\left(p_{n}, q_{n}\right)=\lambda_{1}(p, q)
$$

This completes the proof.

Remark 4.2. Observe that the segment property is used only to prove that

$$
\lambda_{1}(p, q) \leq \liminf _{n \rightarrow+\infty} \lambda_{1}\left(p_{n}, q_{n}\right) .
$$

\section{References}

[1] A. Anane, Simplicité et isolation de la première valeur propre du p-Laplacien avec poids [Simplicity and isolation of the first eigenvalue of the p-Laplacian with weight], C. R. Acad. Sci. Paris Sér. I Math. 305 (1987), no. 16, 725-728 (French).

[2] K. Chaïb, Extension of Díaz-Saás inequality in $\mathbf{R}^{N}$ and application to a system of p-Laplacian, Publ. Mat. 46 (2002), no. 2, 473-488.

[3] F. de Thélin, Sur l'espace propre associé à la première valeur propre du pseudo-Laplacien [Some properties of the eigenfunctions associated with the first eigenvalue of the pseudo-Laplacian], C. R. Acad. Sci. Paris Sér. I Math. 303 (1986), no. 8, 355-358 (French).

[4] _ Première valeur propre d'un système elliptique non linéaire [First eigenvalue of a nonlinear elliptic system], C. R. Acad. Sci. Paris Sér. I Math. 311 (1990), no. 10, 603-606 (French).

[5] J. I. Díaz, Nonlinear Partial Differential Equations and Free Boundaries. Vol. I. Elliptic Equations, Research Notes in Mathematics, vol. 106, Pitman, Massachusetts, 1985.

[6] A. El Khalil, Autour de la première courbe propre du p-Laplacien, Thèse de Doctorat, 1999.

[7] A. El Khalil, P. Lindqvist, and A. Touzani, On the stability of the first eigenvalue of $A_{p} u+$ $\lambda g(x)|u|^{p-2} u=0$ with varying p, Rend. Mat. Appl. (7) 24 (2004), no. 2, 321-336.

[8] A. El Khalil, M. Ouanan, and A. Touzani, Bifurcation of nonlinear elliptic system from the first eigenvalue, Electron. J. Qual. Theory Differ. Equ. 2003 (2003), no. 21, 1-18.

[9] J. Fleckinger, R. F. Manásevich, N. M. Stavrakakis, and F. de Thélin, Principal eigenvalues for some quasilinear elliptic equations on $R^{N}$, Adv. Differential Equations 2 (1997), no. 6, 9811003.

[10] P. Lindqvist, On the equation $\operatorname{div}\left(|\nabla u|^{p-2} \nabla u\right)+\lambda|u|^{p-2} u=0$, Proc. Amer. Math. Soc. 109 (1990), no. 1, 157-164. 
[11] On nonlinear Rayleigh quotients, Potential Anal. 2 (1993), no. 3, 199-218.

[12] J. L. Vázquez, A strong maximum principle for some quasilinear elliptic equations, Appl. Math. Optim. 12 (1984), no. 3, 191-202.

Abdelouahed El Khalil: Département de Mathématiques et Génie Industriel, Ecole Polytechnique de Montréal, Canada H3C 3A7

E-mail address: abdelouahed.el-khalil@polymtl.ca

Said El Manouni: 23 Biesentaler Street, 13359 Berlin, Germany

Current address: Department of Mathematics, Faculty of Sciences Dhar-Mahraz, P.O. Box 1796 Atlas, Fez 30000, Morocco

E-mail address: manouni@hotmail.com

Mohammed Ouanan: Department of Mathematics, Faculty of Sciences Dhar-Mahraz, P.O. Box 1796 Atlas, Fez 30000, Morocco

E-mail address: m_ouanan@hotmail.com 


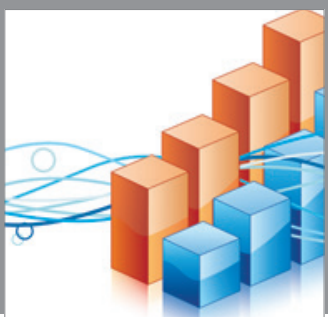

Advances in

Operations Research

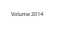

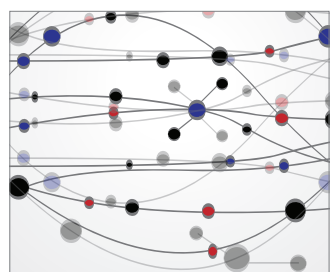

\section{The Scientific} World Journal
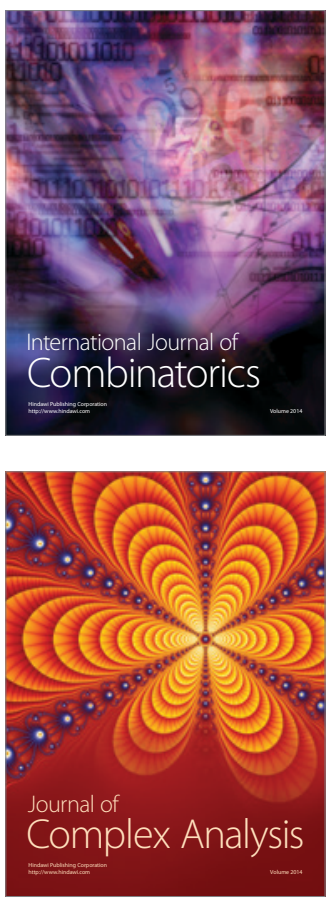

International Journal of

Mathematics and

Mathematical

Sciences
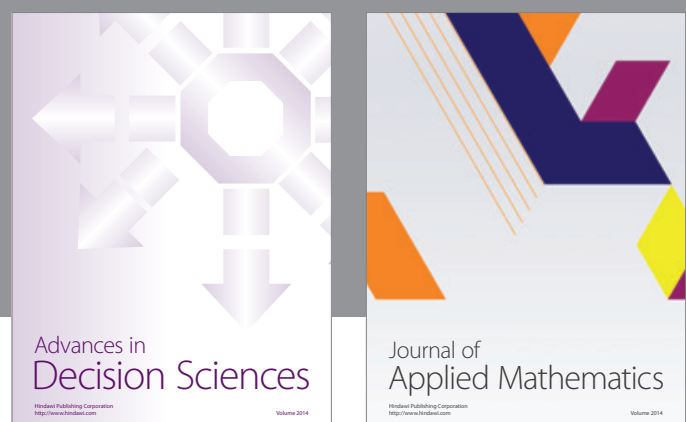

Journal of

Applied Mathematics
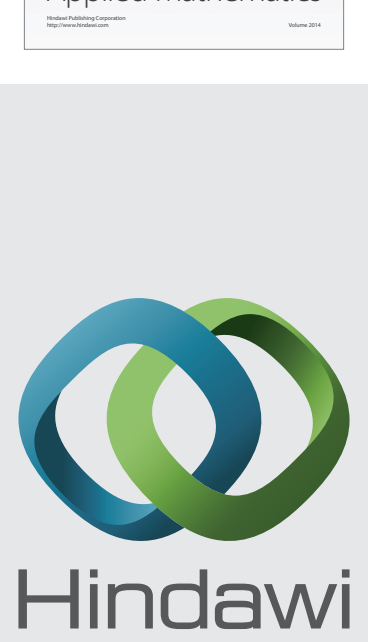

Submit your manuscripts at http://www.hindawi.com
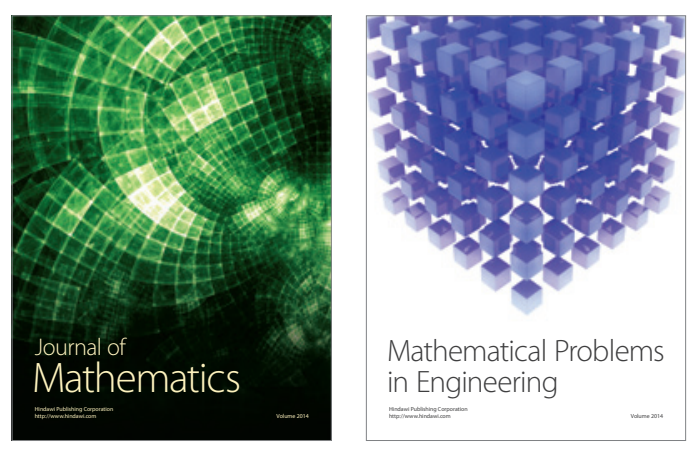

Mathematical Problems in Engineering
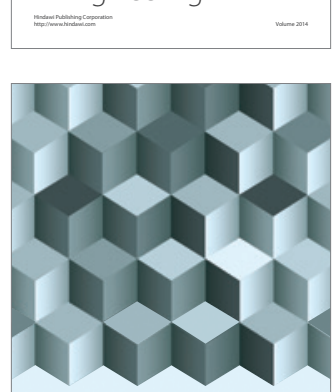

Journal of

Function Spaces
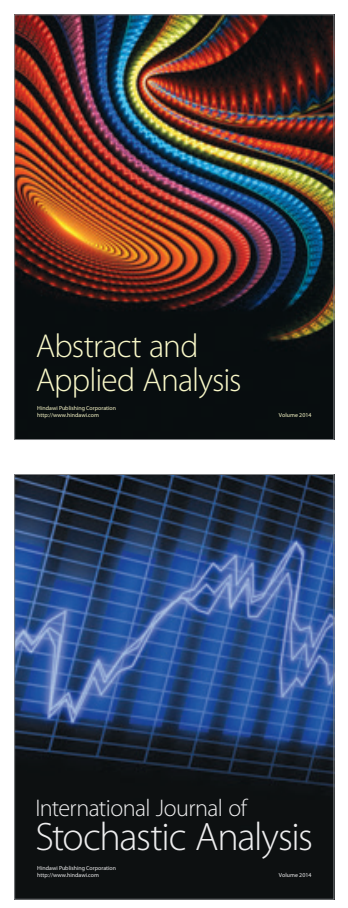

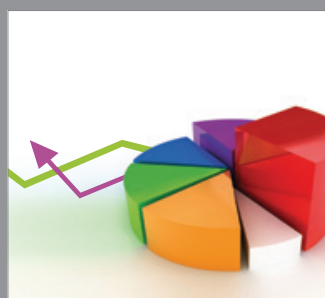

ournal of

Probability and Statistics

Promensencen
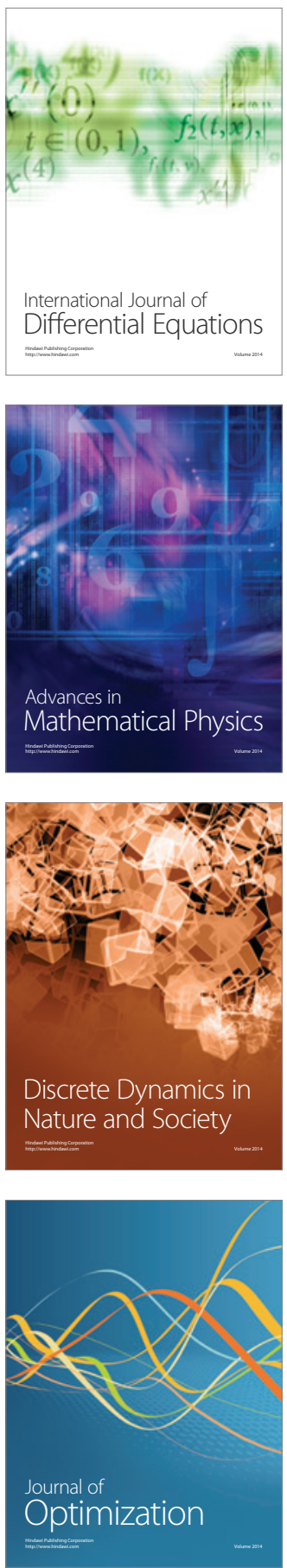